\title{
Boolean Considerations on John Buridan's Octagons of Opposition
}

\author{
Lorenz Demey* \\ ${ }^{*}$ Center for Logic and Philosophy of Science, KU Leuven, Belgium
}

v1.0 released June 2009

\begin{abstract}
This paper studies John Buridan's octagons of opposition for the de re modal propositions and the propositions of unusual construction. Both Buridan himself and the secondary literature have emphasized the strong similarities between these two octagons (as well as a third one, for propositions with oblique terms). In this paper, I argue that the interconnection between both octagons is more subtle than has previously been thought: if we move beyond the Aristotelian relations, and also take Boolean considerations into account, then the strong analogy between Buridan's octagons starts to break down. These differences in Boolean structure can already be discerned within the octagons themselves; on a more abstract level, they lead to these two octagons having different degrees of Boolean complexity (i.e. Boolean closures of different sizes). These results are obtained by means of bitstring analysis, which is one of the key tools from contemporary logical geometry. Finally, I argue that this historical investigation is directly relevant for the theoretical framework of logical geometry, and discuss how it helps us to address certain open questions in this framework.
\end{abstract}

\section{Introduction}

John Buridan's Summulae de Dialectica famously contains three 'octagons of opposition' (Klima 2001, van der Lecq 2005). These octagons can be seen as generalizations or extensions of the well-known square of opposition, from four to eight propositions. One octagon of opposition is concerned with de re modal propositions (e.g. 'every man necessarily runs'), a second one deals with the so-called propositions of unusual construction, in which the predicate precedes the copula (e.g. 'every human some animal is'), and the third one contains propositions with oblique (non-nominative) terms (e.g. 'every donkey of every man runs'). Of these three diagrams, the modal octagon has received by far the most attention in the contemporary literature (Hughes 1987, Lagerlund 2000, 2011, Campos-Benitez 2012, Read 2015b, Johnston 2017), although there has also been some work on the octagon for the propositions of unusual construction (CamposBenitez 2014). Furthermore, Read 2012 discusses all three octagons simultaneously.

Buridan's three octagons are highly similar to each other, as far as the Aristotelian relations are concerned. They all display the same configuration of Aristotelian relations among their eight propositions (this will be made more precise later in the paper). These strong similarities were explicitly noted by Buridan himself; for example, regarding both the propositions of unusual construction and those with oblique terms, he says (Klima 2001, p. 43, my emphases):

from these [combinations] there results a complex figure of eight vertices, and in every vertex there are nine propositions, just as in the figure for modal propositions. And then, consequently, from the eight vertices there result twenty-eight combinations, the oppositions of which have rules entirely similar to those given concerning modal propositions. For in its own way this figure here is similar to the one there

These similarities have also been emphasized in the secondary literature. For example, Read 2012 writes that 'Buridan points to a revealing analogy between the three octagons' and that 'Buridan was able $[\ldots]$ to exhibit a strong analogy between modal, oblique and non-normal [i.e. unusual construction] propositions in his three octagons' (resp. p. 93 and p. 109, my emphases). Further- 
more, in his translation of the Summulae, Klima 2001 notes that 'this reconstruction presents the relationships of all three types of propositions (namely, modals, propositions with oblique terms, and propositions "of unusual construction") that Buridan recognizes as exhibiting the same type of inferential pattern' (p. 45, my emphasis). More recently, Klima 2016 has again noted that 'having observed the analogy of the logical behaviour of dual quantifiers, quantified oblique terms and modal operators, Buridan expanded his Octagon to these further types of propositions as well' (p. 330, my emphasis). ${ }^{1}$

The overarching goal of this paper is to revisit Buridan's octagons, and to argue that their interconnections are more subtle than has previously been thought: if we move beyond the Aristotelian relations, and also take Boolean considerations into account, then the strong analogy between Buridan's octagons starts to break down. In particular, I will show that the Boolean structure of Buridan's modal octagon differs from that of his octagon for the propositions of unusual construction. ${ }^{2}$ These differences in Boolean structure can be discerned within the octagons themselves (in terms of certain propositions in an octagon being logically equivalent to the conjunctions/disjunctions of other propositions in that octagon); on a more abstract level, they also lead to these two octagons having different degrees of Boolean complexity (i.e. Boolean closures of different sizes). Furthermore, I will show that this historical discussion naturally ties in with various issues from logical geometry, i.e. the contemporary, systematic investigation of Aristotelian diagrams. Typical examples of such issues are the existence of Boolean subfamilies within families of Aristotelian diagrams, and the use of bitstrings to measure the Boolean complexity of Aristotelian diagrams.

The paper is organized as follows. Section 2 introduces Buridan's modal octagon, and studies some of its Boolean properties. Similarly, Section 3 introduces Buridan's unusual construction octagon, and compares its Aristotelian and Boolean structures to those of the modal octagon. Next, Section 4 offers a more detailed and systematic investigation of the Boolean differences between the two octagons, making extensive use of bitstring analysis. Section 5 then shows that this historical investigation is directly relevant for the theoretical framework of logical geometry, and discusses how it helps us to address certain open questions in this framework. Finally, Section 6 summarizes the results obtained in this paper, and mentions some questions for future research. ${ }^{3}$

\section{Buridan's modal octagon}

We begin by considering Buridan's modal octagon, which contains eight de re modal propositions. Buridan starts from the four usual categorical statements (from the classical square of opposition), and adds the modalities of necessity and possibility to them. For example, given an ordinary categorical statement of the form 'all $S$ are $P$ ', we can construct the de re modal propositions of the form 'all $S$ are necessarily $P$ ' and 'all $S$ are possibly $P$ '. In this way, Buridan obtains a total number of eight modal propositions, which are listed here (together with a symbolic formalization in the language of first-order modal logic, ${ }^{4}$ and an abbreviation that will be used throughout this paper):

\footnotetext{
${ }^{1}$ Based on these similarities, Campos-Benitez 2014 constructs 'a more abstract Octagon' (p. 362), which yields Buridan's three concrete octagons as specific instances. Each of Buridan's octagons is essentially the result of composing two independent squares of opposition (Demey and Steinkruger 2017), and thus also functions as a duality cube for composed operator duality (Demey 2012, Demey and Smessaert 2016a, Demey and Smessaert 2018c).

${ }^{2}$ I will thus not say much in this paper about Buridan's third octagon of opposition, i.e. the octagon for propositions with oblique terms. Roughly speaking, the idea is that this third octagon is analogous to the modal octagon, not only from an Aristotelian perspective (which was already well-known; cf. the quotations given in the main text), but also from a Boolean perspective (which will be established in this paper; cf. Footnote 19 in particular).

${ }^{3}$ For ease of exposition, I will work with symbolic formalizations of Buridan's propositions (both the modal propositions and those of unusual construction) in the language of contemporary first-order logic. However, it should be emphasized that the overall argumentation presented in this paper does not hinge in any way on the particular details of these symbolic formalizations.

${ }^{4}$ The same formalizations are also used by Read $2015 a$ (p. 40).
} 
1. all $S$ are necessarily $P$

2. all $S$ are possibly $P$

3. some $S$ are necessarily $P$

4. some $S$ are possibly $P$

5. all $S$ are necessarily not $P$

6. all $S$ are possibly not $P$

7. some $S$ are necessarily not $P$

8. $\quad$ some $S$ are possibly not $P$

$\begin{array}{ll}\exists x \diamond S x \wedge \forall x(\diamond S x \rightarrow \square P x) & \forall \square \\ \exists x \diamond S x \wedge \forall x(\diamond S x \rightarrow \diamond P x) & \forall \diamond \\ \exists x(\diamond S x \wedge \square P x) & \exists \square \\ \exists x(\diamond S x \wedge \diamond P x) & \exists \triangleright \\ \forall x(\diamond S x \rightarrow \square \neg P x) & \forall \square \neg \\ \forall x(\diamond S x \rightarrow \diamond \neg P x) & \forall \diamond \neg \\ \neg \exists x \diamond S x \vee \exists x(\diamond S x \wedge \square \neg P x) & \exists \square \neg \\ \neg \exists x \diamond S x \vee \exists x(\diamond S x \wedge \diamond \neg P x) & \exists \diamond \neg\end{array}$

Before moving on, I should first make some remarks about these propositions and their symbolic formalizations. First of all, note that in the formalizations, the subject term $S$ is systematically preceded by a possibility operator $\diamond$. This is meant to capture Buridan's theory of ampliation, which holds that in all modal propositions, the subject term does not merely supposit for actually existing entities, but also for possibly existing entities. For example, Buridan explains that 'all $S$ are possibly $P$ ' should be understood as 'everything which is or can be $S$ is possibly $P^{\prime}$ (Klima 2001, p. 84), which can straightforwardly be formalized as $\forall x((S x \vee \diamond S x) \rightarrow \diamond P x)$ (modulo the issue of existential import; cf. infra). However, since actuality implies possibility, the disjunction $S x \vee \diamond S x$ is logically equivalent to its second disjunct, $\diamond S x$, and thus the entire formalization can be simplified to $\forall x(\diamond S x \rightarrow \diamond P x)$ (again modulo existential import).

Buridan took necessity propositions as well as possibility propositions to ampliate their subject terms. This is by no means uncontroversial; for example, William of Ockham held that possibility propositions ampliate their subject terms, but necessity propositions do not. Hodges and Read's assessment is that 'Ockham is arguably truer to the everyday understanding of modal propositions than Buridan' (Hodges and Read 2010, p. 30). Nevertheless, it is exactly Buridan's 'symmetric' theory of ampliation in modal propositions (both necessity and possibility propositions ampliate their subject terms) that allows him to obtain a well-defined octagon of opposition for them. By contrast, Ockham's 'asymmetric' theory of ampliation leaves him unable to obtain a similarly well-defined octagon. For Ockham, a question such as whether there obtains a relation of contradiction between 'every $S$ is necessarily $P$ ' and 'some $S$ is possibly not $P$ ' is not entirely well-defined, and thus becomes, at best, a matter of interpretation (Hodges and Read 2010, p. 30). ${ }^{5}$ Johnston $2015 b$ provides a more detailed discussion about the debate between Buridan and Ockham regarding ampliation in modal propositions.

Finally, note that Buridan holds that affirmative propositions have existential import, but negative propositions do not (Klima 2009, Ch. 6). Consequently, the first-order formalizations of $\forall \square$ and $\forall \diamond$ contain a conjunct of the form $\exists x \diamond S x$, while those of $\exists \square \neg$ and $\exists \diamond \neg$ contain a disjunct of the form $\neg \exists x \triangleleft S x$. In these conjuncts/disjuncts, too, the subject term $S$ is preceded by a possibility operator $\diamond$, again because of Buridan's theory of ampliation in modal propositions. Note that $\exists x \diamond S x$ expresses that there actually exists an object that possibly has the property $S$. One might reasonably wonder whether this is sufficiently general to capture the full force of Buridan's ampliation theory. After all, maybe there are objects that do not actually exist, yet possibly have the property $S$. However, it has been a matter of some scholarly controversy whether Buridan admitted such bare possibilia (cf. Lagerlund 2000, p. 160, Read 2015a, p. 18, $35 \mathrm{ff}$.), and I will not delve into it further in the present paper. ${ }^{6}$

Buridan goes on to determine the Aristotelian relations holding between these eight de re modal propositions, and finds that 'we have ten subalternations, five contrarieties, five subcontrarieties, four contradictions, and four disparities, which obey no law [of opposition]. And thus

\footnotetext{
${ }^{5}$ Johnston 2015a summarizes the situation as follows: 'Buridan argues that if one wants possibility propositions to ampliate their subjects to the possible, then one must either reject the relationships of contradiction [...] or accept that necessity propositions also ampliate their subjects in the same way' (p. 5). This statement can equivalently be reformulated as follows: assuming that one wants to maintain the relations of contradiction (in order to be able to construct a well-defined octagon of opposition), if one wants possibility propositions to ampliate their subjects to the possible, then one should accept that necessity propositions also ampliate their subjects in the same way.

${ }^{6}$ Thanks to an anonymous referee for some useful discussion about this issue.
} 
(a)

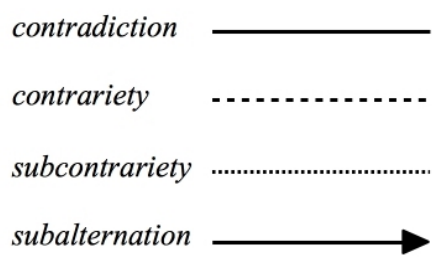

(b)

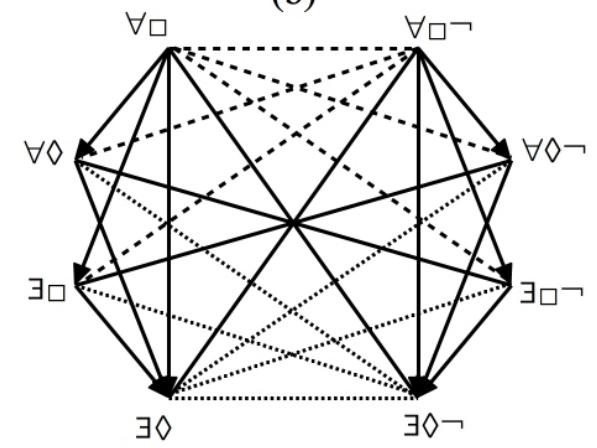

Figure 1. (a) Code for visualizing the Aristotelian relations; (b) Buridan's modal octagon.

clearly there are twenty-eight combinations' (Klima 2001, p. 79). ${ }^{7}$ For example, the propositions $\forall \square$ and $\exists \diamond \neg$ are contradictory: they cannot be true together and they cannot be false together. ${ }^{8}$ Similarly, $\forall \square$ and $\forall \square \neg$ are contrary to each other: these two propositions cannot be true together, but they can be false together. Notably, Buridan also finds four pairs of propositions 'that are disparate, and do not stand in any relation of opposition' (Klima 2001, p. 79), because "such propositions can be true at the same time [...] and they can be false at the same time $[\ldots]$. And [...] it is impossible that one should follow from the other' (Klima 2001, p. 81). ${ }^{9}$ In order to visually represent the eight modal propositions, together with the Aristotelian relations holding between them, Buridan draws an octagon of opposition. Figure 1(b) shows a modern version of this octagon, making use of the abbreviations for the modal propositions introduced above. $^{10}$

The Aristotelian structure of the modal octagon (which propositions stand in which Aristotelian relations?) was already fully described by Buridan. However, neither Buridan himself, nor anyone in the secondary literature, seems to have studied the Boolean structure of this octagon in any detail. That is exactly what we are going to do now.

In some places, the Boolean structure of the octagon is fully determined by its Aristotelian relations. For example, since there is a subalternation from $\forall \square$ to $\forall \diamond$, it follows that the conjunction $\forall \square \wedge \forall \diamond$ is logically equivalent to $\forall \square$ itself, and that the disjunction $\forall \square \vee \forall \diamond$ is logically equivalent to $\forall \diamond$ itself. Similarly, since $\forall \square$ is contrary to $\forall \square \neg$, it follows that the conjunction $\forall \square \wedge \forall \square \neg$ is contradictory $(\perp)$ in nature, and since $\exists \diamond$ is subcontrary to $\exists \diamond \neg$, it follows that the disjunction $\exists \triangleright \vee \exists \triangleright \neg$ is tautological $(\top)$ in nature. ${ }^{11}$ However, in other places, the Boolean structure of the modal octagon is not determined by its Aristotelian relations. For example, since $\forall \diamond$ and $\exists \square$ are unconnected (disparatae), their conjunction and disjunction are contingent propositions, whose relationship to the other propositions in the octagon has to be determined independently. Let us first examine their conjunction, and then their disjunction.

First of all, we consider the relation between $\forall \square$ and the conjunction of $\forall \triangleright$ and $\exists \square$. Since $\forall \square$ entails $\forall \diamond$ as well as $\exists \square$ (cf. the subalternations in the octagon in Figure 1(b)), it follows

\footnotetext{
${ }^{7}$ Given 8 propositions, there are indeed $\frac{8 \times 7}{2}=28$ pairs of distinct propositions, i.e. 28 pairs that can stand in some Aristotelian relation.

${ }^{8}$ Recall the earlier remarks (in particular, Footnote 5) about the debate between Buridan and Ockham regarding ampliation in modal propositions, and its impact on the relations of contradiction.

${ }^{9}$ The third condition can be split into 'the first proposition cannot follow from the second one' and 'the second proposition cannot follow from the first one'. Buridan's characterization of disparatae thus essentially consists of four conditions, viz. two about the propositions being able to be true/false together, and two about the propositions not following from each other. In contemporary logical geometry, Buridan's notion of disparatae is usually called 'independence' or 'unconnectedness', and its four-condition characterization is studied in much more detail (Smessaert and Demey 2014b).

${ }^{10}$ See Read 2012 (p. 106) for a reproduction of Buridan's modal octagon as it actually appears in the manuscripts.

${ }^{11}$ Based on his Tractatus de consequentiis (Hubien 1976), it is clear that Buridan would agree with these Boolean considerations regarding the Aristotelian relations. For example, the seventh conclusion of the treatise's first book reads as follows: 'From every conjunctive proposition consisting of two mutual contradictories any other [proposition] follows in a formal consequence' (Read 2015a, p. 79); in other words: every conjunction of two contradictory propositions is itself contradictory $(\perp)$ in nature. Thanks to Christophe Geudens for bringing this point to my attention.
} 


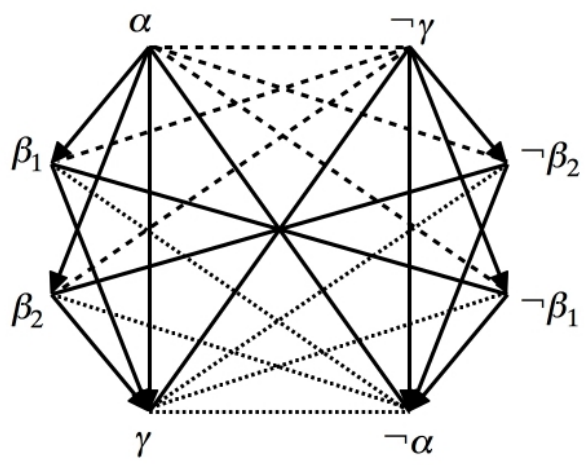

Figure 2. Generic labels for the propositions appearing in Buridan's octagons.

that $\forall \square$ also entails their conjunction, $\forall \diamond \wedge \exists \square$. However, the converse entailment does not hold. To see this, consider a first-order Kripke model $M=\langle W, R, D, I\rangle$, with a set of possible worlds $W=\{w, v\}$, the universal accessibility relation $R=W \times W$, a domain of individuals $D=\{a, b\}$ that is the same for each possible world, and an interpretation function $I$, which is defined as follows: $I(S, w)=\{a, b\}, I(S, v)=\emptyset, I(P, w)=\{a\}$ and $I(P, v)=\{a, b\}$. Since $a \in I(S, w)$, it follows that $M, w \models S a$, which, together with $w R w$, implies that $M, w \models \diamond S a$. Analogously, one can show that $M, w=\diamond S b, M, w \models \square P a, M, w \models \diamond P b$ and $M, w \not \models \square P b$. It now easily follows that $M, w \models \exists x \diamond S x \wedge \forall x(\diamond S x \rightarrow \diamond P x)$, i.e. $M, w \models \forall \diamond$. Similarly, we also have $M, w \models \exists x(\diamond S x \wedge \square P x)$, i.e. $M, w \models \exists \square$. Putting these two results together, we thus have $M, w \mid=\forall \diamond \wedge \exists \square$. However, since $M, w \mid=\diamond S b$ and yet $M, w \forall \vee \square P b$, it follows that $M, w \not \forall \forall x(\diamond S x \rightarrow \square P x)$ and thus also $M, w \not \forall \exists x \diamond S x \wedge \forall x(\diamond S x \rightarrow \square P x)$, i.e. $M, w \not \forall \forall \square$. Hence, there exist a model $M$ and possible world $w$ such that $M, w \models \forall \diamond \wedge \exists \square$, while $M, w \forall \forall \forall \square$, which means exactly that $\forall \diamond \wedge \exists \square$ does not entail $\forall \square$. We thus find that $\forall \square$ entails, but is itself not entailed by, $\forall \diamond \wedge \exists \square$. Consequently, $\forall \square$ is not logically equivalent to $\forall \diamond \wedge \exists \square$.

Secondly, we consider the relation between $\exists \diamond$ and the disjunction of $\forall \diamond$ and $\exists \square$. Since $\forall \diamond$ as well as $\exists \square$ entail $\exists \triangleright$ (cf. the subalternations in the octagon in Figure 1(b)), it follows that their disjunction, $\forall \diamond \vee \exists \square$, also entails $\exists \diamond$. However, the converse entailment does not hold. To see this, consider again a first-order Kripke model $M=\langle W, R, D, I\rangle$, with a set of possible worlds $W=\{w, v\}$, the universal accessibility relation $R=W \times W$, a domain of individuals $D=\{a, b\}$ that is the same for each possible world, and an interpretation function $I$, which is now defined as follows: $I(S, w)=\{a, b\}, I(S, v)=\emptyset, I(P, w)=\{a\}$ and $I(P, v)=\emptyset$. One can easily verify that $M, w \models \diamond S a, M, w=\diamond S b, M, w \models \diamond P a, M, w \not \models \square P a$ and $M, w \not \models \diamond P b$. It now easily follows that $M, w=\exists x(\diamond S x \wedge \diamond P x)$, i.e. $M, w=\exists \diamond$. However, since $M, w \models \diamond S b$ and yet $M, w \not \models \diamond P b$, it follows that $M, w \not \models \forall x(\diamond S x \rightarrow \diamond P x)$ and thus also $M, w \not \forall \exists x \diamond S x \wedge \forall x(\diamond S x \rightarrow \diamond P x)$, i.e. $M, w \not \models \forall \diamond$. Similarly, one can show that $M, w \not \forall \exists x(\diamond S x \wedge \square P x)$, i.e. $M, w \not \models \exists \square$. Putting these last two results together, we thus have $M, w \not \forall \forall \diamond \vee \exists \square$. Hence, there exist a model $M$ and possible world $w$ such that $M, w \models \exists \triangleright$, while $M, w \forall \forall \forall \diamond \vee \exists \square$, which means exactly that $\exists \triangleright$ does not entail $\forall \diamond \vee \exists \square$. We thus find that $\forall \diamond \vee \exists \square$ entails, but is itself not entailed by, $\exists \diamond$. Consequently, $\forall \diamond \vee \exists \square$ is not logically equivalent to $\exists \diamond$.

In sum, then, $\forall \square$ is not logically equivalent to $\forall \diamond \wedge \exists \square$, while $\exists \diamond$ is not logically equivalent to $\forall \diamond \vee \exists \square$. Moving from the left side to the right side of the modal octagon, one can show in exactly the same way that $\forall \square \neg$ is not logically equivalent to $\forall \diamond \neg \wedge \exists \square \neg$, while $\exists \diamond \neg$ is not logically equivalent to $\forall \diamond \neg \vee \exists \square \neg$.

To facilitate our discussion of Buridan's octagons, we will introduce generic labels for the propositions that appear in them. In particular, $\alpha$ will denote the proposition that appears in an octagon's upper left corner, $\beta_{1}$ and $\beta_{2}$ will denote the two 'intermediate' propositions on the left side of the octagon, and $\gamma$ will denote the proposition that appears in the octagon's lower left corner; the negated propositions $\neg \alpha, \neg \beta_{1}, \neg \beta_{2}$ and $\neg \gamma$ systematically appear in the diametrically opposed corners on the right side of the octagon; cf. Figure 2. For example, in Buridan's modal 
octagon, $\alpha$ stands for the concrete modal proposition $\forall \square, \beta_{1}$ and $\beta_{2}$ stand for the concrete modal propositions $\forall \diamond$ and $\exists \square$, and $\gamma$ stands for the concrete modal proposition $\exists \diamond$. Reformulating our earlier conclusions in terms of these generic labels, we find that in Buridan's modal octagon

- $\alpha$ is not logically equivalent to $\beta_{1} \wedge \beta_{2}$,

- $\gamma$ is not logically equivalent to $\beta_{1} \vee \beta_{2}$,

- $\neg \alpha$ is not logically equivalent to $\neg \beta_{1} \vee \neg \beta_{2}$,

- $\neg \gamma$ is not logically equivalent to $\neg \beta_{1} \wedge \neg \beta_{2}$.

From a Boolean perspective, Buridan's modal octagon thus turns out to be highly symmetric in nature. First of all, there is a symmetry between the upper and lower parts of the modal octagon: (i) neither $\alpha$ nor $\gamma$ is logically equivalent to the conjunction/disjunction of $\beta_{1}$ and $\beta_{2}$, and (ii) neither $\neg \alpha$ nor $\neg \gamma$ is logically equivalent to the conjunction/disjunction of $\neg \beta_{1}$ and $\neg \beta_{2}$. Secondly, there is a symmetry between the left and right parts of the modal octagon: (i) neither $\alpha$ nor $\neg \gamma$ is logically equivalent to the conjunction of $(\neg) \beta_{1}$ and $(\neg) \beta_{2}$, and (ii) neither $\gamma$ nor $\neg \alpha$ is logically equivalent to the disjunction of $(\neg) \beta_{1}$ and $(\neg) \beta_{2}$.

\section{Buridan's octagon for the propositions of unusual construction}

We now turn to another of Buridan's octagons, viz. that for the propositions of unusual construction. In such propositions, the predicate precedes the copula; furthermore, the copula expresses identity, ${ }^{12}$ and both the subject term and the predicate term are explicitly quantified, in order to indicate whether or not they are distributed. This constitutes a typical example of the tendency in late medieval logic to use the language of Latin in a highly regimented form (Klima 2016, Read 2017). Buridan again starts from the four usual categorical statements (from the classical square of opposition), and puts the predicate term in front of the copula, together with a quantifier (all/some). For example, given an ordinary categorical statement of the form 'all $S$ are $P$ ', we can construct propositions of unusual construction of the form 'all $S$ all $P$ are' and 'all $S$ some $P$ are'. In this way, Buridan obtains a total number of eight propositions of unusual construction, which are listed here (together with a symbolic formalization in the language of first-order logic with identity, and an abbreviation that will be used throughout this paper) (Klima 2016, p. 330):

1. all $S$ all $P$ are

2. all $S$ some $P$ are

3. some $S$ all $P$ are

4. some $S$ some $P$ are

5. all $S$ all $P$ are not

6. all $S$ some $P$ are not

7. some $S$ all $P$ are not

8. some $S$ some $P$ are not

$$
\begin{array}{ll}
\exists x S x \wedge \exists y P y \wedge \forall x(S x \rightarrow \forall y(P y \rightarrow x=y)) & \forall \forall \\
\exists x S x \wedge \forall x(S x \rightarrow \exists y(P y \wedge x=y)) & \forall \exists \\
\exists y P y \wedge \exists x(S x \wedge \forall y(P y \rightarrow x=y)) & \exists \forall \\
\exists x(S x \wedge \exists y(P y \wedge x=y)) & \exists \exists \\
\forall x(S x \rightarrow \forall y(P y \rightarrow x \neq y)) & \forall \forall \neg \\
\neg \exists y P y \vee \forall x(S x \rightarrow \exists y(P y \wedge x \neq y)) & \forall \exists \neg \\
\neg \exists x S x \vee \exists x(S x \wedge \forall y(P y \rightarrow x \neq y)) & \exists \forall \neg \\
\neg \exists x S x \vee \neg \exists y P y \vee \exists x(S x \wedge \exists y(P y \wedge x \neq y)) & \exists \exists \neg
\end{array}
$$

Recall that Buridan holds that affirmative propositions have existential import, but negative propositions do not (Klima 2009, Ch. 6). Consequently, the first-order formalizations of $\forall \forall$, $\forall \exists$ and $\exists \forall$ contain conjuncts of the form $\exists x S x$ and/or $\exists y P y$, while those of $\forall \exists \neg, \exists \forall \neg$ and $\exists \exists \neg$ contain disjuncts of the form $\neg \exists x S x$ and/or $\neg \exists y P y$. Some of the propositions of unusual construction are logically equivalent to the ordinary categorical statements. In particular, it is straightforward to show that (i) $\forall \exists$ is equivalent to $\exists x S x \wedge \forall x(S x \rightarrow P x)$, i.e. 'all $S$ are $P$ ' (with existential import), (ii) $\exists \exists$ is equivalent to $\exists x(S x \wedge P x)$, i.e. 'some $S$ are $P$ ', (iii) $\exists \forall \neg$ is equivalent to $\neg \exists x S x \vee \exists x(S x \wedge \neg P x)$, i.e. 'not all $S$ are $P$ ' (without existential import), and (iv) $\forall \forall \neg$ is equivalent to $\forall x(S x \rightarrow \neg P x)$, i.e. 'no $S$ are $P$ ' (without existential import). By contrast,

${ }^{12}$ Cf. Malcolm 1979 and Klima 2008 (p. 413) for more details on the identity theory of predication. 
(a)

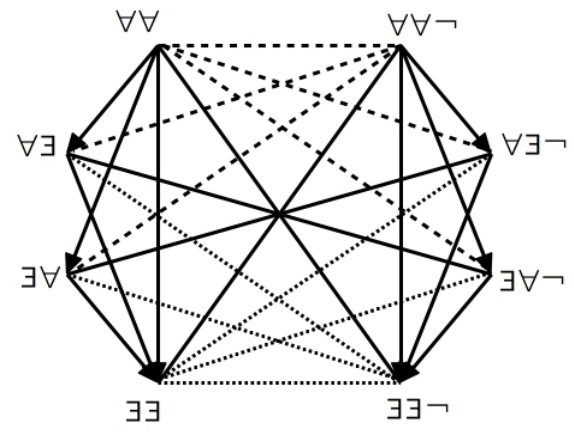

(b)

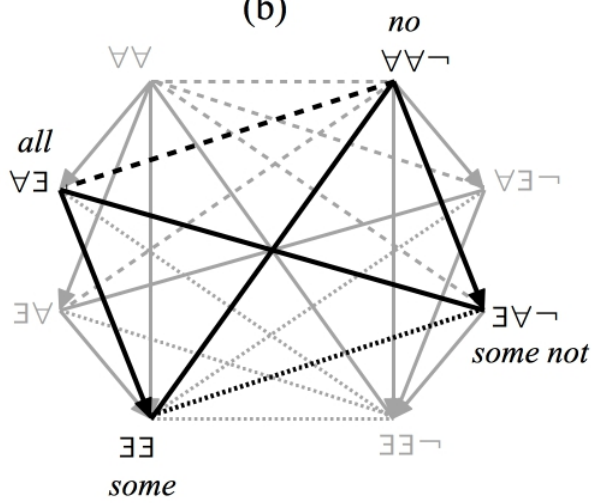

Figure 3. (a) Buridan's octagon for the propositions of unusual construction; (b) the classical square of opposition for the categorical statements embedded as a subdiagram inside this octagon.

the four remaining propositions of unusual construction, $\forall \forall, \exists \forall, \exists \exists \neg$ and $\forall \exists \neg$, are not logically equivalent to any simpler expressions.

Once again, we can determine the Aristotelian relations holding between these eight propositions of unusual construction, and find the same numbers of subalternations, contrarieties, etc. as with the eight de re modal propositions in Figure 1(b). For example, the propositions $\forall \forall$ and $\exists \exists \neg$ are contradictory: they cannot be true together and they cannot be false together. Similarly, $\forall \forall$ and $\forall \forall \neg$ are contrary to each other: these two propositions cannot be true together, but they can be false together. Furthermore, we again find four unconnected pairs of propositions, which do not stand in any Aristotelian relation at all. In order to visually represent the eight propositions of unusual construction, together with the Aristotelian relations holding between them, Buridan draws another octagon of opposition. Figure 3(a) shows a modern version of this octagon, making use of the abbreviations for the propositions of unusual construction introduced above. ${ }^{13}$ Note that the four propositions of unusual construction that are logically equivalent to the categorical statements, i.e. $\forall \exists, \exists \exists, \exists \forall \neg$ and $\forall \forall \neg$, jointly constitute a classical square of opposition, which can be seen as a subdiagram that is embedded inside this octagon; cf. Figure 3(b). More importantly, note that we can again use the labels introduced in Figure 2 to talk about this octagon; for example, $\alpha$ stands for the concrete proposition of unusual construction $\forall \forall, \beta_{1}$ and $\beta_{2}$ stand for the concrete propositions of unusual construction $\forall \exists$ and $\exists \forall$, and $\gamma$ stand for the concrete proposition of unusual construction $\exists \exists$.

The Aristotelian structure of the unusual construction octagon (which propositions stand in which Aristotelian relations?) was already fully described by Buridan. By comparing the unusual construction octagon (Figure 3(a)) to the modal octagon (Figure 1(b)), we immediately see that both diagrams have exactly the same configuration of Aristotelian relations among their propositions. (As was mentioned in the introduction, Buridan himself was already well aware of this.) To make this more precise, we define a bijective function $f$ that maps each modal proposition onto a proposition of unusual construction, by putting $f(\forall \square):=\forall \forall, f(\forall \diamond):=\forall \exists$, $f(\exists \square):=\exists \forall, f(\exists \triangleright):=\exists \exists$, etc. Given any propositions $\varphi, \psi$ from the modal octagon, it can be shown that $\varphi$ and $\psi$ stand in some Aristotelian relation (in the system of first-order modal logic) iff $f(\varphi)$ and $f(\psi)$ stand in that same Aristotelian relation (in the system of first-order logic with identity). Using terminology from logical geometry (Demey 2018, Demey and Smessaert 2018a), the function $f$ is therefore said to be an Aristotelian isomorphism. The octagons in Figures 1(b) and 3(a) thus belong to the same 'family' of Aristotelian diagrams. Since John Buridan was, historically speaking, the first author to study concrete members of this Aristotelian family, it is often called the family of Buridan octagons. ${ }^{14}$

\footnotetext{
${ }^{13}$ See Read 2012 (p. 100) for a reproduction of Buridan's unusual construction octagon as it actually appears in the manuscripts.

14 Just to be clear: the Aristotelian family of Buridan octagons is an abstract class, which contains (infinitely) many
} 
We will now study the Boolean structure of the unusual construction octagon (which has hitherto not been done). Just like with the modal octagon, the Boolean structure of this diagram is in some places entirely determined by its Aristotelian relations. For example, since there is a subalternation from $\forall \forall$ to $\forall \exists$, it follows that the conjunction $\forall \forall \wedge \forall \exists$ is logically equivalent to $\forall \forall$ itself, and that the disjunction $\forall \forall \vee \forall \exists$ is logically equivalent to $\forall \exists$ itself. Similarly, since $\forall \forall$ is contrary to $\forall \forall \neg$, it follows that the conjunction $\forall \forall \wedge \forall \forall \neg$ is contradictory $(\perp)$ in nature, and since $\exists \exists$ is subcontrary to $\exists \exists \neg$, it follows that the disjunction $\exists \exists \vee \exists \exists \neg$ is tautological ( $\top$ ) in nature. However, in other places, the Boolean structure of the unusual construction octagon is not determined by its Aristotelian relations. For example, since $\forall \exists$ and $\exists \forall$ are unconnected (disparatae), their conjunction and disjunction are contingent propositions, whose relationship to the other propositions in the octagon has to be determined independently. Let us first examine their conjunction, and then their disjunction.

First of all, we consider the relation between $\forall \forall$ and the conjunction of $\forall \exists$ and $\exists \forall$. Since $\forall \forall$ entails $\forall \exists$ as well as $\exists \forall$ (cf. the subalternations in the octagon in Figure 3(a)), it follows that $\forall \forall$ also entails their conjunction, $\forall \exists \wedge \exists \forall$. In sharp contrast with the discussion in the previous section, the converse entailment holds as well. To see this, consider an arbitrary first-order model $M=\langle D, I\rangle$ and assume that $M=\forall \exists \wedge \exists \forall$; we will prove that $M \models \forall \forall$. Since $M \models \forall \exists$, it follows that $M \models \exists x S x$; similarly, since $M \models \exists \forall$, it follows that $M \models \exists y P y$. In order to prove that $M \models \forall \forall$, i.e. that $M \models \exists x S x \wedge \exists y P y \wedge \forall x(S x \rightarrow \forall y(P y \rightarrow x=y))$, it thus suffices to show that $M \models \forall x(S x \rightarrow \forall y(P y \rightarrow x=y))$. Consider an arbitrary individual $a \in D$ and assume that $M \models S a$; furthermore, consider an arbitrary individual $b \in D$ and assume that $M \models P b{ }^{15}$ It now suffices to show that $M \models a=b$. From $M \models \exists \forall$ it follows that there exists an individual $c \in D$ such that $M \models S c$ and $M \models \forall x(P x \rightarrow c=x)(\dagger)$. From $M \models \forall \exists$ it follows that $M \models \forall x(S x \rightarrow P x)$. Combining this with (†), we find that $M \models \forall x(S x \rightarrow c=x)(\ddagger)$. Since $M \models S a$, it follows from ( $\ddagger$ ) that $M \models c=a$. Similarly, since $M \models P b$, it follows from ( $\dagger$ ) that $M=c=b$. Since $M \models c=a$ as well as $M \models c=b$, it follows that $M \models a=b$, QED. ${ }^{16}$ We thus find that $\forall \forall$ entails, and is itself entailed by, $\forall \exists \wedge \exists \forall$. Consequently, $\forall \forall$ is logically equivalent to $\forall \exists \wedge \exists \forall$.

Secondly, we consider the relation between $\exists \exists$ and the disjunction of $\forall \exists$ and $\exists \forall$. Since $\forall \exists$ as well as $\exists \forall$ entail $\exists \exists$ (cf. the subalternations in the octagon in Figure 3(a)), it follows that their disjunction, $\forall \exists \vee \exists \forall$, also entails $\exists \exists$. However, the converse entailment does not hold. To see this, consider a first-order model $M=\langle D, I\rangle$ with a domain of individuals $D=\{a, b, c\}$ and an interpretation function $I$, which is defined as follows: $I(S)=\{a, b, c\}$ and $I(P)=\{a, c\}$. One can easily verify that $M \models \exists x(S x \wedge \exists y(P y \wedge x=y))$, i.e. $M \models \exists \exists$. However, since $M \models S b$ and yet $M \not \models P b$, i.e. $M \not \forall \exists y(P y \wedge b=y)$, it follows that $M \not \forall \forall x(S x \rightarrow \exists y(P y \wedge x=y))$ and thus also $M \not \models \exists x S x \wedge \forall x(S x \rightarrow \exists y(P y \wedge x=y))$, i.e. $M \not \forall \forall \exists$. Furthermore, since $a$ and $c$ are two distinct individuals such that $M \mid=P a$ and $M \mid=P c$, it follows that $M \forall \vDash \exists x(S x \wedge \forall y(P y \rightarrow x=y))$ and

diagrams. Some (specifically: three) members of this family were studied by the historical author John Buridan, but it also contains (infinitely) many members that were unknown to Buridan in the Middle Ages. (We will encounter some of these other members in Section 5.) There is nothing paradoxical about this situation. For example, in abstract algebra, the class of Abelian groups is named after the mathematician Niels Abel (1802-1829), and contains (infinitely) many groups. Some members of this class were studied by the historical author Niels Abel, but it also contains (infinitely) many members that were unknown to Abel in the early 19th century.

${ }^{15}$ If desired, this part of the proof can easily be made more precise in the usual model-theoretic way, i.e. by means of assignment functions $g:$ Var $\rightarrow D$ (which map each variable of the object language onto an individual in the domain of the model), the notion of one assignment function $g^{\prime}$ being an $x$-variant (for $x \in \operatorname{Var}$ ) of another assignment function $g$ (i.e. $g \sim_{x} g^{\prime}$ ), etc.

${ }^{16}$ At first sight, it might seem strange that there is a discrepancy between the modal propositions $\forall \square / \forall \diamond / \exists \square$ on the one hand, and the propositions of unusual construction $\forall \forall / \forall \exists / \exists \forall$ on the other hand, since the modalities of necessity ( $\square$ ) and possibility $(\diamond)$ can themselves be understood in terms of resp. universal $(\forall)$ and existential $(\exists)$ quantification over possible worlds. However, the key difference between both types of propositions does not have to do with modalities versus quantifiers, but rather with the fact that the propositions of unusual construction crucially involve the relation of $i d e n t i t y$ (and its property of transitivity). For example, in the proof that $\forall \forall$ is entailed by $\forall \exists \wedge \exists \forall$, we show that two individuals $a$ and $b$ are identical to each other $(a=b)$, by introducing a third individual $c$ and showing that $a$ and $b$ are both identical to it $(a=c$ and $c=b)$. By contrast, the relation of identity (and its property of transitivity) is completely absent from the modal propositions, and thus a similar proof strategy is not available if one were to attempt to prove that $\forall \square$ is entailed by $\forall \diamond \wedge \exists \square$. Thanks to an anonymous referee for some useful discussion about this point. 
thus also $M \not \models \exists y P y \wedge \exists x(S x \wedge \forall y(P y \rightarrow x=y))$, i.e. $M \not \forall \exists \forall$. Putting these last two results together, we thus have $M \not \forall \forall \exists \vee \exists \forall$. Hence, there exists a model $M$ such that $M \models \exists \exists$, while $M \forall \forall \forall \exists \vee \exists \forall$, which means exactly that $\exists \exists$ does not entail $\forall \exists \vee \exists \forall$. We thus find that $\forall \exists \vee \exists \forall$ entails, but is itself not entailed by, $\exists \exists$. Consequently, $\forall \exists \vee \exists \forall$ is not logically equivalent to $\exists \exists$.

In sum, then, $\forall \forall$ is logically equivalent to $\forall \exists \wedge \exists \forall$, while $\exists \exists$ is not logically equivalent to $\forall \exists \vee \exists \forall$. Moving from the left side to the right side of the unusual construction octagon, one can show in exactly the same way that $\forall \forall \neg$ is not logically equivalent to $\forall \exists \neg \wedge \exists \forall \neg$, while $\exists \exists \neg$ is logically equivalent to $\forall \exists \neg \vee \exists \forall \neg$. Reformulating everything in terms of the generic labels introduced above, we find that in Buridan's unusual construction octagon

- $\alpha$ is logically equivalent to $\beta_{1} \wedge \beta_{2}$,

- $\gamma$ is not logically equivalent to $\beta_{1} \vee \beta_{2}$,

- $\neg \alpha$ is logically equivalent to $\neg \beta_{1} \vee \neg \beta_{2}$,

- $\neg \gamma$ is not logically equivalent to $\neg \beta_{1} \wedge \neg \beta_{2}$.

From a Boolean perspective, Buridan's unusual construction octagon is thus not at all symmetric. First of all, there is no symmetry between the upper and lower parts of the unusual construction octagon: (i) $\alpha$ is logically equivalent to the conjunction of $\beta_{1}$ and $\beta_{2}$, but $\gamma$ is not logically equivalent to their disjunction; (ii) $\neg \gamma$ is not logically equivalent to the conjunction of $\neg \beta_{1}$ and $\neg \beta_{2}$, but $\neg \alpha$ is logically equivalent to their disjunction. Secondly, there is no symmetry between the left and right parts of the unusual construction octagon: (i) $\alpha$ is logically equivalent to the conjunction of $\beta_{1}$ and $\beta_{2}$, but $\neg \gamma$ is not logically equivalent to the conjunction of $\neg \beta_{1}$ and $\neg \beta_{2}$; (ii) $\gamma$ is not logically equivalent to the disjunction of $\beta_{1}$ and $\beta_{2}$, but $\neg \alpha$ is logically equivalent to the disjunction of $\neg \beta_{1}$ and $\neg \beta_{2}$.

Let's compare these conclusions to the ones reached at the end of Section 2. It should be clear that, even though Buridan's modal octagon (Figure 1(b)) and his unusual construction octagon (Figure 3(a)) are highly similar in terms of the Aristotelian relations holding between their respective propositions (cf. the Aristotelian isomorphism $f$ introduced above), there are nevertheless subtle Boolean differences between both diagrams. In particular, in the modal octagon, $\alpha$ is not logically equivalent to $\beta_{1} \wedge \beta_{2}$, but in the unusual construction octagon, this equivalence does hold. Furthermore, the modal octagon is highly symmetric (upper/lower and left/right parts) in terms of its Boolean structure, but in the unusual construction octagon, this symmetry is completely lost. Finally, these Boolean differences also have an impact on the Aristotelian isomorphism $f$ between the two octagons. We have already seen that $f$ respects the octagons' Aristotelian structure: $\varphi$ and $\psi$ stand in some Aristotelian relation iff $f(\varphi)$ and $f(\psi)$ stand in that same Aristotelian relation. However, $f$ does not respect the octagons' Boolean structure: we have $\forall \diamond \wedge \exists \square \not \equiv \forall \square$, and yet $f(\forall \diamond) \wedge f(\exists \square)=\forall \exists \wedge \exists \forall \equiv \forall \forall=f(\forall \square)$.

\section{A bitstring analysis of the two octagons}

In the previous two sections I have shown that there are Boolean differences between Buridan's modal octagon and his unusual construction octagon. These differences can already be discerned in the diagrams themselves, for example by asking whether the conjunction of two propositions in a given octagon is logically equivalent to some other proposition in that octagon. In this section I will undertake a more detailed investigation of the Boolean differences between the two octagons. For this purpose I will make use of bitstring analysis, a powerful tool from contemporary logical geometry (Smessaert and Demey 2017).

Bitstrings are combinatorial representations of propositions that provide a concrete grip on the logical behavior of a given Aristotelian diagram (including its Aristotelian relations as well as its Boolean structure). A systematic technique for assigning bitstrings to any finite fragment $\mathcal{F}$ of propositions in any logical system $\mathrm{S}$ is described in detail in Demey and Smessaert 2018a; here I will focus on those aspects that are relevant for our current purposes. Given a fragment $\mathcal{F}=\left\{\varphi_{1}, \ldots, \varphi_{m}\right\}$ from the language $\mathcal{L}_{\mathcal{S}}$ of the logical system $\mathrm{S}$, the partition of $\mathrm{S}$ induced by $\mathcal{F}$ 
is defined as

$$
\Pi_{\mathrm{S}}(\mathcal{F}):=\left\{\alpha \in \mathcal{L}_{\mathrm{S}} \mid \alpha \equiv_{\mathrm{S}} \pm \varphi_{1} \wedge \cdots \wedge \pm \varphi_{m}, \text { and } \alpha \text { is S-consistent }\right\}
$$

(where $+\varphi=\varphi$ and $-\varphi=\neg \varphi$ ). ${ }^{17}$ Furthermore, the Boolean closure of $\mathcal{F}$ in $\mathrm{S}$, denoted $\mathrm{B}_{\mathrm{S}}(\mathcal{F})$, is the smallest Boolean subalgebra of the Lindenbaum-Tarski algebra of $S$ that contains $\mathcal{F}$. Informally, $\mathrm{B}_{\mathrm{S}}(\mathcal{F})$ contains exactly the Boolean combinations of propositions from $\mathcal{F}$. It can be shown that every proposition in the Boolean closure of $\mathcal{F}$ is logically equivalent to a disjunction of elements of the partition induced by $\mathcal{F}$ : for every $\varphi \in \mathrm{B}_{\mathrm{S}}(\mathcal{F})$ we have

$$
\varphi \equiv \mathrm{S} \quad \bigvee\left\{\alpha \in \Pi_{\mathrm{S}}(\mathcal{F}) \mid \models_{\mathrm{S}} \alpha \rightarrow \varphi\right\}
$$

The bitstring semantics $\beta_{\mathrm{S}}^{\mathcal{F}}$ maps each formula $\varphi \in \mathrm{B}_{\mathrm{S}}(\mathcal{F})$ to its bitstring representation $\beta_{\mathrm{S}}^{\mathcal{F}}(\varphi) \in$ $\{0,1\}^{\left|\Pi_{S}(\mathcal{F})\right|}$, which 'keeps track' of which formulas of $\Pi_{S}(\mathcal{F})$ enter into this disjunction. For example, if $\Pi_{\mathrm{S}}(\mathcal{F})=\left\{\alpha_{1}, \alpha_{2}, \alpha_{3}, \alpha_{4}\right\}$, then $\beta_{\mathrm{S}}^{\mathcal{F}}(\varphi)=1011$ means that $\varphi \equiv_{\mathrm{S}} \alpha_{1} \vee \alpha_{3} \vee \alpha_{4}$. Note that $\left|\Pi_{\mathrm{S}}(\mathcal{F})\right|$ is the length of the bitstring $\beta_{\mathrm{S}}^{\mathcal{F}}(\varphi)$. It can be shown that $\beta_{\mathrm{S}}^{\mathcal{F}}$ is an Aristotelian isomorphism between $\mathrm{B}_{\mathrm{S}}(\mathcal{F})$ and $\{0,1\}^{\left|\Pi_{\mathrm{s}}(\mathcal{F})\right|}$, in the sense introduced above. Furthermore, it can also be shown that this mapping is a Boolean algebra isomorphism, and hence, that $\mathrm{B}_{\mathrm{S}}(\mathcal{F})$ is isomorphic to the Boolean algebra $\{0,1\}\}^{\left|\Pi_{s}(\mathcal{F})\right|}$, and thus contains $2^{\left|\Pi_{s}(\mathcal{F})\right|}$ elements. The bitstring length $\left|\Pi_{S}(\mathcal{F})\right|$ thus provides a direct measure of the Boolean complexity of $\mathcal{F}$.

After this (admittedly very quick) summary of bitstring analysis, we are now in a position to apply this technique to Buridan's octagons. We start with the modal octagon. Let $\mathcal{F}_{m}$ be the set of propositions appearing in this octagon, i.e. $\mathcal{F}_{m}:=\{\forall \square, \forall \diamond, \exists \square, \exists \diamond, \forall \square \neg, \forall \diamond \neg, \exists \square \neg, \exists \diamond \neg\}$ (cf. Figure 1(b)), and let FOL ${ }^{\square}$ be some system of first-order modal logic. ${ }^{18}$ In order to determine the partition of $\mathrm{FOL}^{\square}$ that is induced by $\mathcal{F}_{m}$, we have to consider all conjunctions of (possibly negated) propositions from $\mathcal{F}_{m}$. For example, two of these conjunctions are the following:

- $\forall \square \wedge \forall \diamond \wedge \exists \square \wedge \exists \diamond \wedge \neg \forall \square \neg \wedge \neg \forall \diamond \neg \wedge \neg \exists \square \neg \wedge \neg \exists \diamond \neg$ and

- $\forall \square \wedge \neg \forall \diamond \wedge \exists \square \wedge \exists \diamond \wedge \neg \forall \square \neg \wedge \neg \forall \diamond \neg \wedge \neg \exists \square \neg \wedge \neg \exists \diamond \neg$.

The former conjunction can be shown to be $\mathrm{FOL}^{\square}$-equivalent to the simpler proposition $\forall \square$, while the latter can be shown to be $\mathrm{FOL}^{\square}$-inconsistent (because its first two conjuncts are already $\mathrm{FOL}^{\square}$-inconsistent with each other). By systematically going through all conjunctions of this form, rewriting the conjunctions as simpler, $\mathrm{FOL}^{\square}$-equivalent propositions whenever possible, and discarding the $\mathrm{FOL}^{\square}$-inconsistent conjunctions, we find the partition induced by Buridan's modal octagon:

$$
\begin{aligned}
& \Pi_{\mathrm{FOL}}^{\square}\left(\mathcal{F}_{m}\right)= \begin{cases}\alpha_{1} & :=\forall \square, \\
\alpha_{2} & :=\forall \diamond \wedge \exists \square \wedge \exists \diamond \neg,\end{cases} \\
& \alpha_{3}:=\forall \diamond \wedge \forall \diamond \neg, \\
& \alpha_{4}:=\quad \exists \square \wedge \exists \square \neg, \\
& \alpha_{5}:=\forall \diamond \neg \wedge \exists \square \neg \wedge \exists \diamond, \\
& \left.\alpha_{6}:=\forall \square \neg \quad\right\} .
\end{aligned}
$$

For ease of notation, we will write the bitstring semantics $\beta_{\mathrm{FOL}}^{\mathcal{F}_{m}}$ that corresponds to this partition simply as $\beta_{m}$. Since $\left|\Pi_{\mathrm{FOL}} \square\left(\mathcal{F}_{m}\right)\right|=6$, the modal octagon can be represented by means of bitstrings of length 6. For example, since $\exists \square$ is $\mathrm{FOL}^{\square}$-equivalent to $\alpha_{1} \vee \alpha_{2} \vee \alpha_{4}$, it can be

\footnotetext{
${ }^{17}$ The set $\Pi_{\mathrm{S}}(\mathcal{F})$ is called a 'partition' of (the class of models of) $\mathrm{S}$ because its elements are (i) jointly exhaustive, i.e. $\mathrm{S} \models$ $\bigvee \Pi_{\mathrm{S}}(\mathcal{F})$, and (ii) mutually exclusive, i.e. $\mathrm{S} \models \neg(\alpha \wedge \beta)$ for $\operatorname{distinct} \alpha, \beta \in \Pi_{\mathrm{S}}(\mathcal{F})$.

${ }^{18}$ The precise details of $\mathrm{FOL}^{\square}$ do not matter here (as long as it contains the axiom $\square p \rightarrow \diamond p$ ). For example, since $\mathcal{F}_{m}$ only contains de re modal propositions, it is irrelevant whether $\mathrm{FOL}^{\square}$ contains the Barcan formula and/or converse Barcan formula as an axiom (Fitting and Mendelsohn 1998).
} 

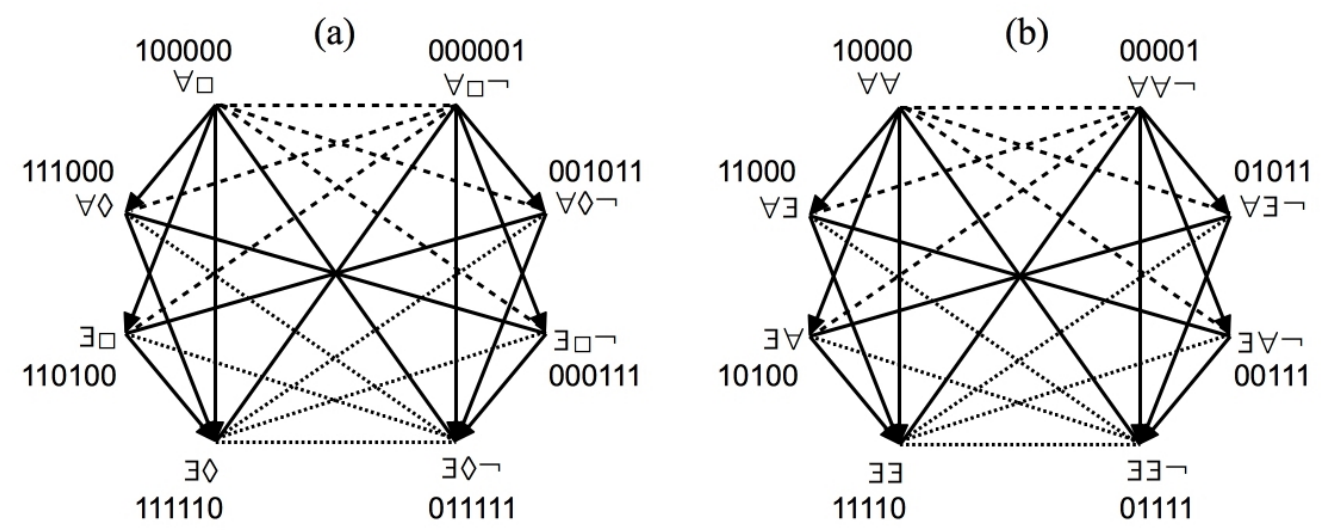

Figure 4. The bitstring representations of Buridan's (a) modal octagon and (b) unusual construction octagon.

represented as the bitstring 110100 , i.e. $\beta_{m}(\exists \square)=110100$. The bitstrings of all eight propositions in the modal octagon are shown in Figure 4(a). In particular, note that

- $\beta_{m}(\forall \diamond) \wedge \beta_{m}(\exists \square)=111000 \wedge 110100=110000 \neq 100000=\beta_{m}(\forall \square)$ and

- $\beta_{m}(\forall \diamond) \vee \beta_{m}(\exists \square)=111000 \vee 110100=111100 \neq 111110=\beta_{m}(\exists \diamond)$.

These are the 'bitstring versions' of the facts that $\forall \diamond \wedge \exists \square$ is not equivalent to $\forall \square$ and that $\forall \diamond \vee \exists \square$ is not equivalent to $\exists \diamond$ (which were originally proved in Section 2). Furthermore, since $\left|\Pi_{\mathrm{FOL}} \square\left(\mathcal{F}_{m}\right)\right|=6$, it follows that the Boolean closure of Buridan's modal octagon is isomorphic to $\{0,1\}^{6}$, i.e. $\mathrm{B}_{\mathrm{FOL}} \square\left(\mathcal{F}_{m}\right) \cong\{0,1\}^{6}$. This Boolean closure thus contains $2^{6}=64$ elements, i.e. there exist 64 distinct Boolean combinations of the propositions that appear in the modal octagon.

We now turn to Buridan's unusual construction octagon. Let $\mathcal{F}_{u}$ be the set of propositions appearing in this octagon, i.e. $\mathcal{F}_{u}:=\{\forall \forall, \forall \exists, \exists \forall, \exists \exists, \forall \forall \neg, \forall \exists \neg, \exists \forall \neg, \exists \exists \neg\}$ (cf. Figure 3(a)), and let $\mathrm{FOL}^{=}$be the system of first-order logic with identity. We again systematically go through all conjunctions of (possibly negated) propositions from $\mathcal{F}_{u}$, rewrite the conjunctions as simpler, $\mathrm{FOL}=$-equivalent propositions whenever possible, and discard the $\mathrm{FOL}^{=}$-inconsistent conjunctions. In this way we find the partition induced by Buridan's unusual construction octagon (note the odd numbering of the partition's elements, which will be explained later):

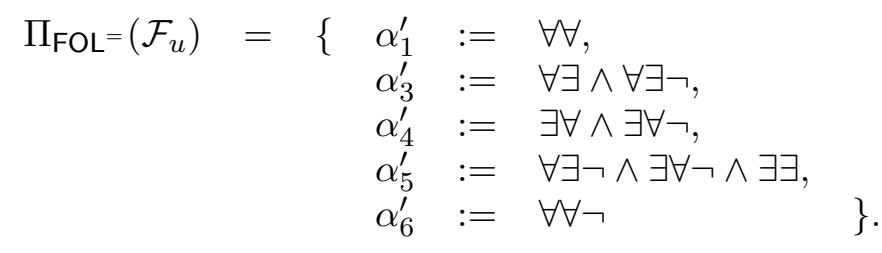

For ease of notation, we will write the bitstring semantics $\beta_{\mathrm{FOL}}^{\mathcal{F}_{u}}=$ that corresponds to this partition simply as $\beta_{u}$. Since $\left|\Pi_{\mathrm{FOL}}=\left(\mathcal{F}_{u}\right)\right|=5$, the unusual construction octagon can be represented by means of bitstrings of length 5 . For example, since $\exists \forall$ is $\mathrm{FOL}^{=}=$-equivalent to $\alpha_{1}^{\prime} \vee \alpha_{4}^{\prime}$, it can be represented as the bitstring 10100, i.e. $\beta_{u}(\exists \forall)=10100$. The bitstrings of all eight propositions in the unusual octagon are shown in Figure 4(b). In particular, note that

- $\beta_{u}(\forall \exists) \wedge \beta_{u}(\exists \forall)=11000 \wedge 10100=10000=\beta_{u}(\forall \forall)$ and

- $\beta_{u}(\forall \exists) \vee \beta_{u}(\exists \forall)=11000 \vee 10100=11100 \neq 11110=\beta_{u}(\exists \exists)$.

These are the 'bitstring versions' of the facts that $\forall \exists \wedge \exists \forall$ is equivalent to $\forall \forall$ and that $\forall \exists \vee \exists \forall$ is not equivalent to $\exists \exists$ (which were originally proved in Section 3). Furthermore, since $\left|\Pi_{\mathrm{FOL}}=\left(\mathcal{F}_{u}\right)\right|=5$, it follows that the Boolean closure of Buridan's unusual construction octagon is isomorphic to $\{0,1\}^{5}$, i.e. $\mathrm{B}_{\mathrm{FOL}}=\left(\mathcal{F}_{u}\right) \cong\{0,1\}^{5}$. This Boolean closure thus contains $2^{5}=32$ elements, i.e. there exist 32 distinct Boolean combinations of the propositions that appear in the unusual 
construction octagon.

In light of this bitstring analysis, we can now draw a systematic comparison between Buridan's modal octagon and his unusual construction octagon. The former induces a partition $\Pi_{\mathrm{FOL}} \square\left(\mathcal{F}_{m}\right)$ of 6 elements (and can thus be represented by bitstrings of length 6 ), whereas the latter induces a partition $\Pi_{\mathrm{FOL}}=\left(\mathcal{F}_{u}\right)$ of only 5 elements (and can thus be represented by bitstrings of length $5)$. In other words, the unusual construction octagon has a lower Boolean complexity than the modal octagon. This lower Boolean complexity manifests itself in a higher number of logical equivalences among Boolean combinations of propositions of unusual construction. As we have seen in Section 3, some of these additional equivalences can already be found within the unusual construction octagon itself. However, the full effects of this higher number of logical equivalences can only be felt at the level of the Boolean closures of the two octagons. The Boolean closure of the modal octagon contains $2^{6}=64$ elements, whereas that of the unusual construction octagon contains only $2^{5}=32$ elements. There are thus fewer Boolean combinations of unusual construction propositions than there are of modal propositions. The reason for this is precisely that there are more logical equivalences among Boolean combinations of unusual construction propositions than there are among Boolean combinations of modal propositions. For example, recall that $\forall \square$ and $\forall \diamond \wedge \exists \square$ are not $\mathrm{FOL}^{\square}$-equivalent and thus count as two distinct Boolean combinations in the Boolean closure of the modal octagon; by contrast, their counterparts $\forall \forall$ and $\forall \exists \wedge \exists \forall$ are $\mathrm{FOL}^{=}$-equivalent and thus count as just a single Boolean combination in the Boolean closure of the unusual construction octagon. ${ }^{19}$

Finally, looking back at the Aristotelian isomorphism $f$, it should be noted that the elements of $\Pi_{\mathrm{FOL}}=\left(\mathcal{F}_{u}\right)$ are clearly the counterparts of those of $\Pi_{\mathrm{FOL}} \square\left(\mathcal{F}_{m}\right)$ :

$$
\begin{aligned}
& \alpha_{1}=\forall \square \\
& \alpha_{3}=\forall \diamond \wedge \forall \diamond \neg \\
& \alpha_{4}=\exists \square \wedge \exists \square \neg \\
& \alpha_{5}=\forall \diamond \neg \wedge \exists \square \neg \wedge \exists \diamond \\
& \alpha_{6}=\forall \square \neg \\
& =f(\forall \square) \text {, } \\
& =f(\forall \diamond) \wedge f(\forall \diamond \neg), \\
& =f(\exists \square) \wedge f(\exists \square \neg), \\
& =f(\forall \diamond \neg) \wedge f(\exists \square \neg) \wedge f(\exists \diamond), \\
& =f(\forall \square \neg) \text {. }
\end{aligned}
$$

If we try to determine the counterpart of $\alpha_{2}$ in the same fashion, we find $f(\forall \diamond) \wedge f(\exists \square) \wedge f(\exists \diamond \neg)$, i.e. $\forall \exists \wedge \exists \forall \wedge \exists \exists \neg$; however, the latter is $\mathrm{FOL}^{=}$-inconsistent (note that it can be rewritten as $\left.(\forall \exists \wedge \exists \forall) \wedge \exists \exists \neg \equiv_{\mathrm{FOL}}=\forall \forall \wedge \neg \forall \forall\right)$. Since $\alpha_{2} \in \Pi_{\mathrm{FOL}}\left(\mathcal{F}_{m}\right)$ does not have a counterpart in $\Pi_{\mathrm{FOL}}=\left(\mathcal{F}_{u}\right)$, the bitstring representations of the unusual construction octagon are exactly the result of systematically deleting the second bit position in the bitstring representations of the modal octagon (cf. Figure 4). This process of deleting one bit position does not have any effect on the octagons' Aristotelian stucture (they are Aristotelian isomorphic!), but as we have seen above, it does have a significant effect on their Boolean structure.

To make this more formally precise, consider the sets $\beta_{m}\left[\mathcal{F}_{m}\right]:=\left\{\beta_{m}(\varphi) \mid \varphi \in \mathcal{F}_{m}\right\} \subseteq\{0,1\}^{6}$ and $\beta_{u}\left[\mathcal{F}_{u}\right]:=\left\{\beta_{u}(\varphi) \mid \varphi \in \mathcal{F}_{u}\right\} \subseteq\{0,1\}^{5}$, and let $f^{\prime}: \beta_{m}\left[\mathcal{F}_{m}\right] \rightarrow \beta_{u}\left[\mathcal{F}_{u}\right]$ be the function that systematically deletes a bitstring's second bit position. One can then show that the diagram below commutes, i.e. $\beta_{u}(f(\varphi))=f^{\prime}\left(\beta_{m}(\varphi)\right)$ for all $\varphi \in \mathcal{F}_{m}$. Furthermore, the function $f^{\prime}$ respects the Aristotelian structure of $\beta_{m}\left[\mathcal{F}_{m}\right]$ and $\beta_{u}\left[\mathcal{F}_{u}\right]$ : two bitstrings $b_{1}, b_{2} \in \beta_{m}\left[\mathcal{F}_{m}\right]$ stand in some Aristotelian relation iff $f^{\prime}\left(b_{1}\right), f^{\prime}\left(b_{2}\right) \in \beta_{u}\left[\mathcal{F}_{u}\right]$ stand in that same Aristotelian relation. However, $f^{\prime}$ does not respect the Boolean structure of $\beta_{m}\left[\mathcal{F}_{m}\right]$ and $\beta_{u}\left[\mathcal{F}_{u}\right]$ : we have $111000 \wedge 110100 \neq$ 100000 , and yet $f^{\prime}(111000) \wedge f^{\prime}(110100)=11000 \wedge 10100=10000=f^{\prime}(100000) .{ }^{20}$

\footnotetext{
19 In exactly the same way as was done here for Buridan's modal octagon and unusual construction octagon, one can also perform a bitstring analysis of Buridan's third octagon of opposition, i.e. that for propositions with oblique terms. That octagon turns out to have a Boolean complexity of 6 (i.e. it can be represented with bitstrings of length 6 , and its Boolean closure contains $2^{6}=64$ elements) (Demey and Smessaert 2018a, Subsection 5.2, in particular Footnote 44), just like the modal octagon studied in this paper. While Buridan's three octagons are all Aristotelian isomorphic to each other, there are thus clear differences between them: the modal octagon and the oblique octagon have a Boolean complexity of 6 , whereas the unusual construction octagon has a Boolean complexity of 5 .

${ }^{20}$ This is the 'bitstring version' of the fact that $\forall \diamond \wedge \exists \square$ is not equivalent to $\forall \square$, and yet $f(\forall \diamond) \wedge f(\exists \square)$ is equivalent to $f(\forall \square)$, which was originally pointed out at the end of Section 3.
} 


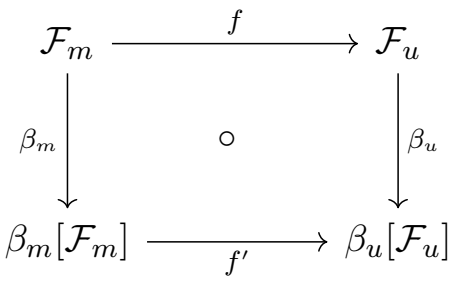

\section{The broader significance for logical geometry}

In recent years it has become clear that Aristotelian diagrams can be fruitfully studied as objects of independent mathematical and philosophical interest, giving rise to the burgeoning field of logical geometry (Demey 2017a, 2018, Demey and Smessaert 2014, 2016b, 2017, 2018a, 2018b, Smessaert and Demey 2014a, 2014b, 2015, 2017). Throughout this paper, we have been using tools and ideas from logical geometry to shed new light on the octagons developed by the medieval logician John Buridan. Typical examples include the notion of unconnectedness (and its four-condition characterization) as a contemporary counterpart to Buridan's disparatae, the notion of an Aristotelian isomorphism to capture the 'strong analogy' between Buridan's octagons, and the idea of one Aristotelian diagram (viz. the classical square of opposition for the categorical statements) being embedded as a subdiagram inside a larger Aristotelian diagram (viz. Buridan's unusual construction octagon). Most importantly, Section 4 was entirely based on bitstring analysis, which is one of the key tools in logical geometry. In the current section I will show that, vice versa, the historical discussion is also directly relevant for the theoretical framework of logical geometry, and that it helps us to address certain open questions in this framework.

One of the crucial insights from logical geometry concerns the interaction between Aristotelian and Boolean structure in Aristotelian diagrams. This insight can be expressed in various ways, depending on which aspect one wishes to emphasize:

- there exist diagrams that are Aristotelian isomorphic, but have different Boolean complexities,

- there exist diagrams that are Aristotelian isomorphic, but are represented by bitstrings of different lengths,

- there exist diagrams that are Aristotelian isomorphic, but have Boolean closures of different sizes,

- there exist Aristotelian diagrams that are Aristotelian isomorphic, but not Boolean isomorphic,

- there exist families of Aristotelian diagrams that have distinct Boolean subfamilies.

The most well-known example of this phenomenon concerns a certain family of Aristotelian hexagons, viz. the so-called Jacoby-Sesmat-Blanché (JSB) hexagons (Demey and Smessaert 2018a, Subsection 5.1). This family is named after Jacoby 1950, Sesmat 1951 and Blanché 1953, 1966, who were the first authors to study this type of hexagon (recall Footnote 14). One can show that the family of JSB hexagons has precisely two Boolean subfamilies: some JSB hexagons have Boolean complexity 3, while others have Boolean complexity 4 . In the literature, the former are often called 'strong JSB hexagons', while the latter are called 'weak JSB hexagons' (Pellissier 2008). Figure 5(a) shows an example of a strong JSB hexagon, which induces the tripartition $\{\square p, \diamond p \wedge \diamond \neg p, \square \neg p\}$ of S5; Figure 5(b) shows an example of a weak JSB hexagon, which induces the quadripartition $\{\square p, p \wedge \diamond \neg p, \neg p \wedge \diamond p, \square \neg p\}$ of $55 .{ }^{21}$ Although the majority of JSB hexagons that appear in the literature have Boolean complexity 3 , one can certainly also find concrete applications of JSB hexagons with Boolean complexity 4. For example, Roelandt

\footnotetext{
${ }^{21}$ Just like with the Buridan octagons, the Boolean differences between the strong and weak JSB hexagons are also manifested within the diagrams themselves. Consider, for example, the three upper propositions in each of the two JSB hexagons in Figure 5. In the strong JSB hexagon, the uppermost proposition ( $\square p \vee \square \neg p)$ is S5-equivalent to the disjunction of the two other upper propositions ( $\square p$ and $\square \neg p$ ). By contrast, in the weak JSB hexagon, the uppermost proposition $(\neg p \vee \square p)$ is not S5-equivalent to the disjunction of the two other propositions $(\square p$ and $\square \neg p)$.
} 
(a)

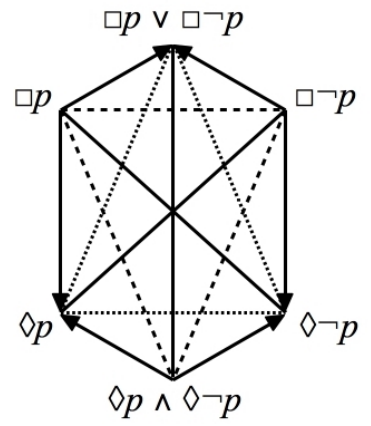

(b)

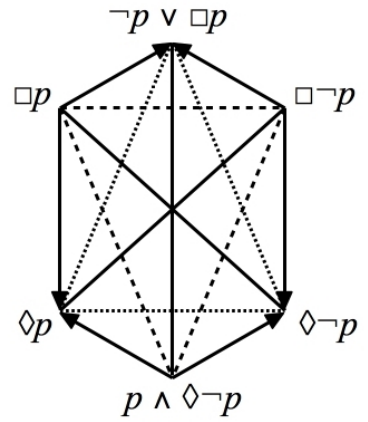

Figure 5. Examples of (a) a strong JSB hexagon and (b) a weak JSB hexagon.

2016 (p. 135ff.) offers several weak JSB hexagons for applications in linguistics.

Another example of this phenomenon concerns the family of Buridan octagons. One can show that this Aristotelian family has precisely three Boolean subfamilies: some Buridan octagons have Boolean complexity 4, others have Boolean complexity 5 , and yet others have Boolean complexity 6 (Demey and Smessaert 2018a, Subsection 5.2). The differences between these three Boolean subfamilies are also manifested within the diagrams themselves. Let's use the generic labels from Figure 2. In Buridan octagons with Boolean complexity $4, \alpha$ is logically equivalent to $\beta_{1} \wedge \beta_{2}$ and $\gamma$ is logically equivalent to $\beta_{1} \vee \beta_{2}$. In Buridan octagons with Boolean complexity 5 , exactly one of these two equivalences holds. Finally, in Buridan octagons with Boolean complexity 6 , neither of these equivalences holds. (Of course, we are already thoroughly familiar with the Boolean differences between Buridan octagons of Boolean complexities 5 and 6, from Sections 2 and 3 of this paper.)

There are some very well-known examples from the extant literature of Buridan octagons with Boolean complexity 6. For example, as we have shown above, Buridan's own modal octagon has Boolean complexity 6. For a more recent example, note that Russell's theory of definite descriptions naturally gives rise to a Buridan octagon with Boolean complexity 6 (Demey 2017b). Similarly, there are some very well-known examples in the extant literature of Buridan octagons with Boolean complexity 4. For example, Figure 6(a) shows a Buridan octagon for classical propositional logic, which has Boolean complexity 4 (Smessaert and Demey 2014a); it can be shown that this diagram induces the quadripartition $\{p \wedge q, p \wedge \neg q, \neg p \wedge q, \neg p \wedge \neg q\}$ of classical propositional logic. Furthermore, Chatti and Schang 2013 and Demey and Smessaert 2016c construct Buridan octagons with Boolean complexity 4 in the context of their investigations on existential import and on metalogic, respectively. In general, and using the generic labels from before, one can prove that if the diagram in Figure 2 is a Buridan octagon with Boolean complexity 6 , then the diagram in Figure 6(b) will be a Buridan octagon with Boolean complexity 4 (note that the Buridan octagon for classical propositional logic in Figure 6(a) is indeed of this form).

The situation is rather different with respect to the Buridan octagons with Boolean complexity 5. Such diagrams can theoretically be proved to exist; furthermore, we can 'artificially' construct as many examples as we want: using the generic labels from before, one can prove that if the diagram in Figure 2 is a Buridan octagon with Boolean complexity 6, then both of the diagrams in Figure 7 will be Buridan octagons with Boolean complexity 5 . However, until now, we did not possess a 'natural' example of this Boolean subfamily, i.e. we had hitherto not found a concrete example of a Buridan octagon with Boolean complexity 5 being used somewhere in the extant literature. This was rather unfortunate, because it seemed to suggest that the classification of the family of Buridan octagons into three distinct Boolean subfamilies is not rooted in the actual applications of Aristotelian diagrams. This would cast significant doubts on the wider methodological significance of this ongoing classificatory effort in logical geometry. 
(a)

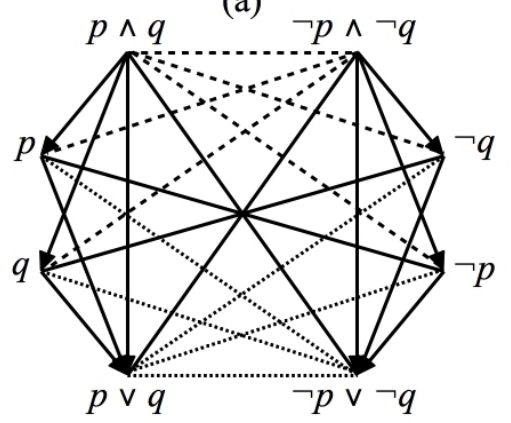

(b)

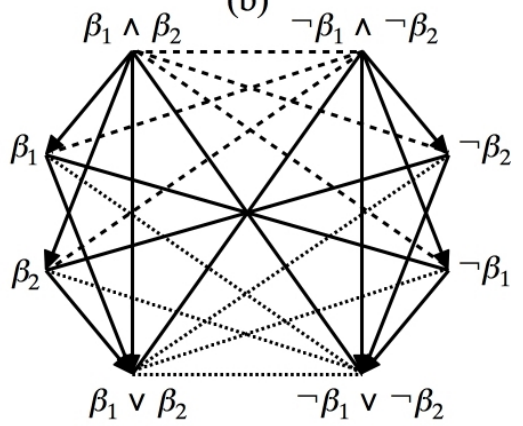

Figure 6. (a) Buridan octagon with Boolean complexity 4 in classical propositional logic, (b) generic Buridan octagon that has Boolean complexity 4, on the assumption that the generic Buridan octagon in Figure 2 has Boolean complexity 6.

(a)

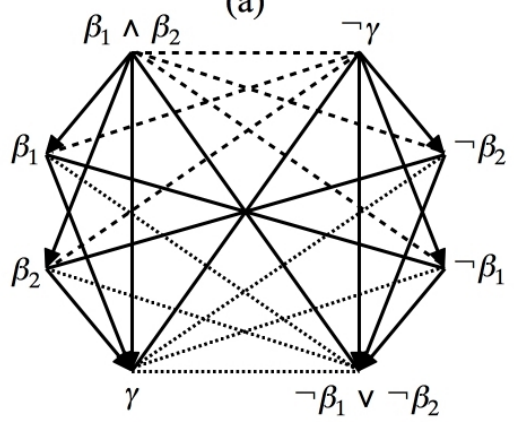

(b)

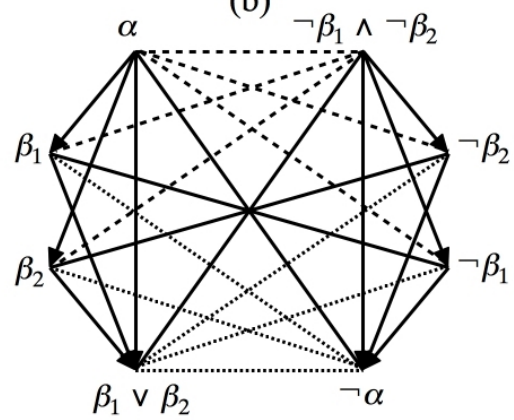

Figure 7. Two generic Buridan octagons that have Boolean complexity 5, on the assumption that the generic Buridan octagon in Figure 2 has Boolean complexity 6.

However, the historical discussion in this paper has delivered - as far as I know for the first time - a concrete example of a Buridan octagon with Boolean complexity 5, viz. John Buridan's very own octagon for the propositions of unusual construction. This shows that the classification of the family of Buridan octagons into three Boolean subfamilies is not merely a theoretical enterprise: each of these three Boolean subfamilies is inhabited by at least one concrete application of an Aristotelian diagram found in the extant literature.

Finally, I would briefly like to reflect on the particular irony of this situation. At first sight, it might seem odd that a natural example of a Buridan octagon with Boolean complexity 5 , which has been sought after for quite some time, is ultimately found in John Buridan himself, i.e. in the very author after which the Aristotelian family is named. This oversight should probably be explained by the fact that Buridan's two other octagons (modal and oblique) have Boolean complexity 6 (cf. Section 2 and Footnote 19), in combination with an overly naïve belief in the 'strong analogy' between all three of Buridan's octagons. This combination of circumstances might have led researchers in logical geometry (including the present author) to tacitly assume that Buridan's unusual construction octagon would also have Boolean complexity 6 , without ever pausing to explicitly check whether this is indeed the case.

\section{Conclusion}

In this paper I have shown that there are subtle Boolean differences between John Buridan's modal octagon and his unusual construction octagon, by making use of tools from contemporary logical geometry (in particular, bitstring analysis). This interaction between historical and theoretical work has proved to be highly fruitful for both sides. On the one hand, the powerful tools from logical geometry allow us to draw a very precise and systematic comparison regarding the Boolean properties of these two octagons. On the other hand, this historical investigation has led to the conclusion that Buridan's unusual construction octagon has Boolean complexity 
5, and thus constitutes the first natural example of this particular Boolean subfamily of the Aristotelian family of Buridan octagons.

This historical/systematic line of research will be continued in future work. For example, Chatti 2014 has recently argued that Avicenna's modal theory gives rise to a dodecagon, which (modulo a difference between de re and de dicto modal propositions) can be seen as a natural extension of Buridan's modal octagon. In order to further substantiate this claim, it might be interesting to use bitstring analysis to investigate the Aristotelian and Boolean properties of this dodecagon (which Aristotelian family does this dodecagon belong to? how many Boolean subfamilies does that Aristotelian family have, and which Boolean complexities do they correspond to? etc.), and to systematically compare them to those of Buridan's modal octagon.

\section{Acknowledgements}

Thanks to Christophe Geudens, Hans Smessaert, Margaux Smets and two anonymous referees for their valuable feedback on an earlier version of this paper. The author holds a Postdoctoral Fellowship of the Research Foundation-Flanders (FWO). The research reported in this paper was partially carried out during a research stay at the Institut für Philosophie II of the RuhrUniversität Bochum, which was financially supported by an FWO travel grant.

\section{References}

Blanché, R. 1953. 'Sur l'opposition des concepts', Theoria, 19, 89-130.

Blanché, R. 1966. Structures Intellectuelles, Paris: Vrin.

Campos-Benítez, J. M. 2012. 'The medieval modal octagon and the S5 Lewis modal system', in: J.-Y. Béziau and G. Payette, eds., The Square of Opposition. A General Framework for Cognition, Bern: Peter Lang, 99-118.

Campos-Benítez, J. M. 2014. 'The medieval octagon of opposition for sentences with quantified predicates', History and Philosophy of Logic, 35, 354-368.

Chatti, S. 2014. 'Avicenna on possibility and necessity', History and Philosophy of Logic, 35, $332-353$.

Chatti, S. and Schang, F. 2013. 'The cube, the square and the problem of existential import', History and Philosophy of Logic, 32, 101-132.

Demey, L. 2012. 'Algebraic aspects of duality diagrams', in: P. T. Cox, B. Plimmer, and P. Rodgers, eds., Diagrammatic Representation and Inference, LNCS 7352, Berlin: Springer, 300-302.

Demey, L. 2017a. 'Using syllogistics to teach metalogic', Metaphilosophy, 48, 575-590.

Demey, L. 2017b. 'The logical geometry of Russell's theory of definite descriptions', unpublished manuscript.

Demey, L. 2018. 'Computing the maximal Boolean complexity of families of Aristotelian diagrams', Journal of Logic and Computation, 28, 1323-1339.

Demey, L. and Smessaert, H. 2014. 'The relationship between Aristotelian and Hasse diagrams', in: T. Dwyer, H. Purchase, and A. Delaney, eds., Diagrammatic Representation and Inference, LNCS 8578, Berlin: Springer, 213-227.

Demey, L. and Smessaert, H. 2016a. 'Duality in logic and language', in: J. Fieser and B. Dowden, eds., Internet Encyclopedia of Philosophy, Martin: University of Tennessee, 1-37.

Demey, L. and Smessaert, H. 2016b. 'The interaction between logic and geometry in Aristotelian diagrams', in: M. Jamnik, Y. Uesaka, and S. Elzer Schwartz, eds., Diagrammatic Representation and Inference, LNCS 9781, Berlin: Springer, 67-82.

Demey, L. and Smessaert, H. 2016c. 'Metalogical decorations of logical diagrams', Logica Universalis, 10, 233-292.

Demey, L. and Smessaert, H. 2017. 'Logical and geometrical distance in polyhedral Aristotelian diagrams in knowledge representation', Symmetry, 9(10), 204. 
Demey, L. and Smessaert, H. 2018a. 'Combinatorial bitstring semantics for arbitrary logical fragments', Journal of Philosophical Logic, 47, 325-363.

Demey, L. and Smessaert, H. 2018b. 'Geometric and cognitive differences between Aristotelian diagrams for the Boolean algebra $\mathrm{B}_{4}$ ', Annals of Mathematics and Artificial Intelligence, $\mathbf{8 3}$, $185-208$.

Demey, L. and Smessaert, H. 2018c. 'Aristotelian and duality relations beyond the square of opposition', in: P. Chapman, G. Stapleton, A. Moktefi, S. Perez-Kriz, and F. Bellucci, eds., Diagrammatic Representation and Inference, LNCS 10871, Berlin: Springer, 640-656.

Demey, L. and Steinkrüger, P. 2017. 'De logische geometrie van Johannes Buridanus' modale achthoek', Tijdschrift voor Filosofie, 79, 217-238.

Fitting, M. and Mendelsohn, R. L. 1998. First-Order Modal Logic, Dordrecht: Kluwer.

Hodges, W. and Read, S. 2010. 'Western logic', Journal of the Indian Council of Philosophical Research, XXVII, 13-45.

Hubien, H. 1976. Iohannis Buridani Tractatus de consequentiis, Philosophes Médiévaux XVI, Louvain: Publications universitaires.

Hughes, G. E. 1987. 'The modal logic of John Buridan', in: G. Corsi, C. Mangione, and M. Mugnai, eds., Atti del convegno internazionale di storia della logica, le teorie delle modalità, Bologna: CLUEB, 93-111.

Jacoby, P. 1950. 'A triangle of opposites for types of propositions in Aristotelian logic', New Scholasticism, 24, 32-56.

Johnston, S. 2015a. 'A formal reconstruction of Buridan's modal syllogism', History and Philosophy of Logic, 36, 2-17.

Johnston, S. 2015b. 'Ockham and Buridan on the ampliation of modal propositions', British Journal for the History of Philosophy, 23, 234-255.

Johnston, S. 2017. 'The modal octagon and John Buridan's modal ontology', in: J.-Y. Béziau and G. Basti, eds., The Square of Opposition: A Cornerstone of Thought, Berlin: Springer, $35-52$.

Klima, G. 2001. John Buridan, Summulae de Dialectica, New Haven: Yale University Press.

Klima, G. 2008. 'The nominalist semantics of Ockham and Buridan: A "rational reconstruction", in: D. M. Gabbay and J. Woods, eds., Handbook of the History of Logic. Volume 2: Mediaeval and Renaissance Logic, Amsterdam: Elsevier, 389-431.

Klima, G. 2009. John Buridan, Oxford: Oxford University Press.

Klima, G. 2016. 'Consequence', in: C. Dutilh Novaes and S. Read, eds., The Cambridge Companion to Medieval Logic, Cambridge: Cambridge University Press, 316-341.

Lagerlund, H. 2000. Modal Syllogistics in the Middle Ages, Leiden: Brill.

Lagerlund, H. 2011. 'Theories of syllogism', in: H. Lagerlund, ed., Encyclopedia of Medieval Philosophy, Berlin: Springer, 1236-1241.

Malcolm, J. 1979. 'A reconsideration of the identity and inherence theories of the copula', Journal of the History of Philosophy, 17, 383-400.

Pellissier, R. 2008. 'Setting n-opposition', Logica Universalis, 2, 235-263.

Read, S. 2012. 'John Buridan's theory of consequence and his octagons of opposition', in: J.-Y. Béziau and D. Jacquette, eds., Around and Beyond the Square of Opposition, Berlin: Springer, 93-110.

Read, S. 2015a. John Buridan: Treatise on Consequences, New York: Fordham University Press.

Read, S. 2015b. 'John Buridan on non-contingency syllogisms', in: A. Koslow and A. Buchsbaum, eds., The Road to Universal Logic. Volume 1, Berlin: Springer, 447-456.

Read, S. 2017. 'Non-normal propositions in Buridan's logic', in: L. Cesalli, F. Goubier, and A. De Libera, eds., Formal Approaches and Natural Language in Medieval Logic, Turnhout: Brepols, 453-468.

Roelandt, K. 2016. The Meaning of Most. Proportional and Comparative Interpretations in Dutch, Utrecht: LOT Publications.

Sesmat, A. 1951. Logique II. Les Raisonnements. La syllogistique, Paris: Hermann. 
Smessaert, H. and Demey, L. 2014a. 'Logical and geometrical complementarities between Aristotelian diagrams', in: T. Dwyer, H. Purchase, and A. Delaney, eds., Diagrammatic Representation and Inference, LNCS 8578, Berlin: Springer, 246-260.

Smessaert, H. and Demey, L. 2014b. 'Logical geometries and information in the square of opposition', Journal of Logic, Language and Information, 23, 527-565.

Smessaert, H. and Demey, L. 2015. 'Béziau's contributions to the logical geometry of modalities and quantifiers', in: A. Koslow and A. Buchsbaum, eds., The Road to Universal Logic, Vol. I, Berlin: Springer, 475-493.

Smessaert, H. and Demey, L. 2017. 'The unreasonable effectiveness of bitstrings in logical geometry', in: J.-Y. Béziau and G. Basti, eds., The Square of Opposition: A Cornerstone of Thought, Berlin: Springer, 197-214.

van der Lecq, R. 2005. Johannes Buridanus: Summulae de Propositionibus, Artistarium 10-1, Turnhout: Brepols. 